\title{
AUTHOR INDEX \\ Volume 5 (2007)
}

\author{
Abnizova, I., Walter, K., te Boekhorst, R., Elgar, G. and Gilks, \\ W. R., Statistical Information Characterization of Conserved \\ Non-Coding Elements in Vertebrates
}

2b (2007) 533

Akberdin, I. R., Ozonov, E. A., Mironova, V. V., Omelyanchuk,

N. A., Likhoshvai, V. A., Gorpinchenko, D. N. and Kolchanov,

N. A., A Cellular Automaton to Model the Development of

Primary Shoot Meristems of Arabidopsis Thaliana

2b (2007) 641

Alexov, E., see Kundrotas, P.

3 (2007) 693

Almabrazi, H., see O'Leary, B. M.

6 (2007) 1155

Andorf, J., see O'Leary, B. M.

6 (2007) 1155

Andries, E., Hagstrom, T., Atlas, S. R. and Willman, C.,

Regularization Strategies for Hyperplane Classifiers:

Application to Cancer Classification with Gene

Expression Data

1 (2007) 79

Archakov, A. I., see Ivanov, A. S.

2b (2007) 579

Arnold, J., see Tewari, S.

2a (2007) 201

Asai, K., see Sakakibara, Y.

5 (2007) 1103

Atlas, S. R., see Andries, E.

1 (2007) 79

Baker, C. J. O., see Kanagasabai, R.

6 (2007) 1319

Baker, C. J. O., see Witte, R.

6 (2007) 1339

Bar-Hen, A., Kim, K. I. and van de Wiel, M. A., Some

Comments on False Discovery Rate

4 (2007) 987

Barb, A. S., see Shyu, C.-R.

6 (2007) 1193

Barrera, J., see Stransky, B.

4 (2007) 977

Baumgartner, Jr., W. A., see Caporaso, J. G.

6 (2007) 1233

Ben-Gal, I., see Posch, S.

2b (2007) 561

Bent, E., see Fu, Q.

4 (2007) 937

2b (2007) 593

Bezmaternykh, K. D., see Mishchenko, E. L.

2a (2007) 201

Bhandarkar, S. M., see Tewari, S.

$1(2007) 47$

Motif-Finding in Biopolymer Sequences

Bicego, M., Dellaglio, F. and Felis, G. E., Multimodal

Phylogeny for Taxonomy: Integrating Information

from Nucleotide and Amino Acid Sequences

5 (2007) 1069 
Bickerton, G. R. J., see Worth, C. L.

6 (2007) 1297

Blundell, T. L., see Worth, C. L.

6 (2007) 1297

Bodén, M., see Suksawatchon, J.

1 (2007) 117

Boeva, V., see Tsiporkova, E.

5 (2007) 1005

Borneman, J., see Fu, Q.

4 (2007) 937

Braun, T. A., see O'Leary, B. M.

6 (2007) 1155

Broach, J. R., see Tan, M. P.

4 (2007) 895

Brown, B., see O'Leary, B. M.

6 (2007) 1155

Bryant, D. H., see Chen, B. Y.

2a (2007) 353

$\mathrm{Bu}, \mathrm{D}$., see $\mathrm{Yu}, \mathrm{C}$.

2a (2007) 297

Burke, D. F., see Worth, C. L.

6 (2007) 1297

Burns, T., see O'Leary, B. M.

6 (2007) 1155

Cai, J., see Yu, C.

2a (2007) 297

Caporaso, J. G., Baumgartner, Jr., W. A., Randolph, D. A., Cohen, K. B. and Hunter, L., Rapid Pattern Development for Concept Recognition Systems: Application to Point Mutations

6 (2007) 1233

Casavant, T. L., see O'Leary, B. M.

6 (2007) 1155

Chan, K. C. C., see Ma, P. C. H.

3 (2007) 651

Chen, B. Y., Bryant, D. H., Fofanov, V. Y., Kristensen, D. M., Cruess, A. E., Kimmel, M., Lichtarge, O. and Kavraki, L. E., Cavity Scaling: Automated Refinement of Cavity-Aware Motifs in Protein Function Prediction

Chen, J., see Xie, M.

2a (2007) 353

3 (2007) 795

Chen, J. Y., Yan, Z., Shen, C., Fitzpatrick, D. P. G. and Wang,

M., A Systems Biology Approach to the Study of Cisplatin

Drug Resistance in Ovarian Cancers

Chen, R., see Yu, C.

2a (2007) 383

2a (2007) 297

Cheng, T. M. K., see Worth, C. L.

6 (2007) 1297

Chia, K. S., see Mah, J. T. L.

5 (2007) 1123

Choo, K. H., see Kanagasabai, R.

6 (2007) 1319

Christova, P., see Kundrotas, P.

3 (2007) 693

Chrobak, M., see Fu, Q.

4 (2007) 937

Cios, K. J., see Nguyen, C.

3 (2007) 739

Clark, A. F., see O'Leary, B. M.

6 (2007) 1155

Coe, E., see Shyu, C.-R.

6 (2007) 1193

Cohen, K. B., see Caporaso, J. G.

6 (2007) 1233

Comet, J.-P., see Mateus, D.

2b (2007) 627

Cruess, A. E., see Chen, B. Y.

2a (2007) 353

Daraselia, N., see Sivachenko, A. Y.

2b (2007) 429

Davis, S. G., see O'Leary, B. M.

6 (2007) 1155

de Ridder, D., see van Berlo, R. J. P.

4 (2007) 839

de Souza, S. J., see Stransky, B.

4 (2007) 977 
Dellaglio, F., see Bicego, M.

5 (2007) 1069

Deronne, K. W. and Karypis, G., Effective Optimization

Algorithms for Fragment-Assembly Based Protein

Structure Prediction

2a (2007) 335

2b (2007) 611

Efremov, R. G., see Polyansky, A. A.

2b (2007) 533

Elgar, G., see Abnizova, I.

Erdogmus, M. and Sezerman, O. U., Application of Automatic

Mutation-Gene Pair Extraction to Diseases

6 (2007) 1261

Ermakova, E. O., Nurtdinov, R. N. and Gelfand, M. S.,

Overlapping Alternative Donor Splice Sites

in the Human Genome

5 (2007) 991

Eskin, E. and Snir, S., Incorporating Homologues into Sequence Embeddings for Protein Analysis

3 (2007) 717

Fadeev, S. I., see Gunbin, K. V.

2b (2007) 491

5 (2007) 1069

2a (2007) 281

Feng, L., see Han, X.

Fisher, W. G., Rosenblatt, K. P., Fishman, D. A., Whiteley,

G. R., Mikulskis, A., Kuzdzal, S. A., Lopez, M. F., Tan,

N. C., German, D. C. and Garner, H. R., A Robust

Biomarker Discovery Pipeline for High-Performance Mass

Spectrometry Data

Fishman, D. A., see Fisher, W. G.

5 (2007) 1023

5 (2007) 1023

2a (2007) 383

4 (2007) 895

6 (2007) 1277

2a (2007) 353

6 (2007) 1297

Forman, J. R., see Worth, C. L.

Friberg, M. T., Prediction of Transcription Factor Binding Sites Using ChIP-Chip and Phylogenetic Footprinting Data

1 (2007) 105

3 (2007) 773

6 (2007) 1277

Friedrich, C. M., see Klinger, R.

Fu, Q., Bent, E., Borneman, J., Chrobak, M. and Young, N. E., Algorithmic Approaches to Selecting Control Clones in DNA Array Hybridization Experiments

Fu, Z. and Jiang, T., Computing the Breakpoint Distance

Between Partially Ordered Genomes

5 (2007) 1087

Furlong, L. I., see Klinger, R.

6 (2007) 1277

Gallois, J.-P., see Mateus, D.

2b (2007) 627

Gardiner, K. J., see Nguyen, C.

3 (2007) 739

Garner, H. R., see Fisher, W. G.

5 (2007) 1023

Gelfand, M. S., see Ermakova, E. O.

5 (2007) 991

Georgieva, P., see Kundrotas, P.

3 (2007) 693

German, D. C., see Fisher, W. G.

5 (2007) 1023 
Gilks, W. R., see Abnizova, I.

2b (2007) 533

Glazko, G., see Klebanov, L.

5 (2007) 1139

Gnedenko, O. V., see Ivanov, A. S.

2b (2007) 579

Gohr, A., see Posch, S.

2b (2007) 561

Gong, S., see Worth, C. L.

6 (2007) 1297

González-Vélez, H., see González-Vélez, V.

3 (2007) 755

González-Vélez, V. and González-Vélez, H., Parallel Stochastic

Simulation of Macroscopic Calcium Currents

3 (2007) 755

Gorpinchenko, D. N., see Akberdin, I. R.

2b (2007) 641

Grau, J., see Posch, S.

2b (2007) 561

Green, J., see Shyu, C.-R.

6 (2007) 1193

Grosse, I., see Posch, S.

2b (2007) 561

Grundstad, J. A., see O'Leary, B. M.

6 (2007) 1155

Gunbin, K. V., Omelyanchuk, L. V., Kogai, V. V., Fadeev, S. I. and Kolchanov, N. A., Model of the Reception of Hedgehog Morphogen Concentration Gradient: Comparison with an Extended Range of Experimental Data

2b (2007) 491

Gusfield, D., see Wu, Y.

Haavisto, O., Hyötyniemi, H. and Roos, C., State Space Modeling of Yeast Gene Expression Dynamics

Hagstrom, T., see Andries, E.

Han, X., Sung, W.-K. and Feng, L., Identifying Differentially Expressed Genes in Time-Course Microarray Experiment Without Replicate

Harnsomburana, J., see Shyu, C.-R.

Hawkins, T. and Kihara, D., Function Prediction of

Uncharacterized Proteins

2a (2007) 181

1 (2007) 31

1 (2007) 79

Hebbel, R. P., see Jiang, A.

Heida, N., see Masuya, H.

Hofestädt, R., Extended Backus-Systems for the Representation and Specification of the Genome

Hofmann-Apitius, M., see Klinger, R.

(2007) 457

Huang, H.-C., see Li, W.

6 (2007) 1277

2a (2007) 251

Hunter, L., see Caporaso, J. G.

6 (2007) 1233

Hyötyniemi, H., see Haavisto, O.

1 (2007) 31

Ivanisenko, V. A., see Mishchenko, E. L.

2b (2007) 593

Ivanov, A. S., Gnedenko, O. V., Molnar, A. A., Mezentsev,

Y. V., Lisitsa, A. V. and Archakov, A. I., Protein-Protein

Interactions as New Targets for Drug Design: Virtual

and Experimental Approaches

2b (2007) 579

Jiang, A., Pan, W., Milbauer, L. C., Shyr, Y. and Hebbel, R. P., A Practical Question Based on Cross-Platform Microarray 
Data Normalization: Are BOEC More Like Large Vessel or Microvascular Endothelial Cells or Neither of Them?

Jiang, T., see Fu, Z.

Kaburagi, T., Muramatsu, D. and Matsumoto, T.,

Transmembrane Structure Predictions with Hydropathy

Index/Charge Two-Dimensional Trajectories of Stochastic

Dynamical Systems

Kachko, A. V., see Khlebodarova, T. M.

Kadarmideen, H. N., see von Rohr, P.

Kanagasabai, R., Choo, K. H., Ranganathan, S. and Baker,

C. J. O., A Workflow for Mutation Extraction and Structure Annotation

Karypis, G., see Deronne, K. W.

Kavraki, L. E., see Chen, B. Y.

Kazic, T., see Shyu, C.-R.

Kel, A. E., see Posch, S.

Kemp, M. B., see O'Leary, B. M.

Khlebodarova, T. M., see Mishchenko, E. L.

Khlebodarova, T. M., Tikunova, N. V., Kachko, A. V.,

Stepanenko, I. L., Podkolodny, N. L. and Kolchanov, N. A.,

Application of Bioinformatics Resources for Genosensor

Design

Kihara, D., see Hawkins, T.

Kim, J. H., see Park, T.

Kim, K., see Park, T.

Kim, K. I., see Bar-Hen, A.

Kimmel, M., see Chen, B. Y.

Klebanov, L., Glazko, G., Salzman, P., Yakovlev, A. and Xiao,

Y., A Multivariate Extension of the Gene Set Enrichment

Analysis

Klinger, R., Friedrich, C. M., Mevissen, H. T., Fluck, J.,

Hofmann-Apitius, M., Furlong, L. I. and Sanz, F., Identifying

Gene-Specific Variations in Biomedical Text

Kogai, V. V., see Gunbin, K. V.

Kolchanov, N. A., see Akberdin, I. R.

Kolchanov, N. A., see Gunbin, K. V.

Kolchanov, N. A., see Khlebodarova, T. M.

Kolchanov, N. A., see Mishchenko, E. L.

Kristensen, D. M., see Chen, B. Y.

Kundrotas, P., Georgieva, P., Shosheva, A., Christova, P. and

Alexov, E., Assessing the Quality of the Homology-Modeled

3D Structures from Electrostatic Standpoint: Test on

Bacterial Nucleoside Monophosphate Kinase Families
$4(2007) 875$

5 (2007) 1087

3 (2007) 669

2b (2007) 507

3 (2007) 773

6 (2007) 1319

2a (2007) 335

2a (2007) 353

6 (2007) 1193

2b (2007) 561

6 (2007) 1155

2b (2007) 593

2b (2007) 507

1 (2007) 1

4 (2007) 865

4 (2007) 865

4 (2007) 987

2a (2007) 353

5 (2007) 1139

6 (2007) 1277

2b (2007) 491

2b (2007) 641

2b (2007) 491

2b (2007) 507

2b (2007) 593

2a (2007) 353

3 (2007) 693 
Kuzdzal, S. A., see Fisher, W. G.

5 (2007) 1023

Lachenal, N., see Yip, Y. L.

6 (2007) 1215

Le Gall, P., see Mateus, D.

2b (2007) 627

Lee, S., see Park, T.

4 (2007) 865

Lee, S., see Worth, C. L.

6 (2007) 1297

Lee, Y.-S., see Park, T.

4 (2007) 865

Leontiev, V., see O'Leary, B. M.

6 (2007) 1155

Li, G., Lu, J., Olman, V. and Xu, Y., Prediction of Cis-Regulatory Elements: From High-Information Content Analysis to Motif Identification

$4(2007) 817$

Li, W., Peng, Y., Huang, H.-C. and Liu, Y., Biomarker Discovery and Visualization in Gene Expression Data with Efficient Generalized Matrix Approximations

Lichtarge, O., see Chen, B. Y.

Likhoshvai, V. A., see Akberdin, I. R.

Likhoshvai, V. A., see Mishchenko, E. L.

Likhoshvai, V. and Ratushny, A., Generalized Hill Function Method for Modeling Molecular Processes

Lin, G., see Wan, X.

Lin, Y., see $\mathrm{Yu}, \mathrm{C}$.

Lisitsa, A. V., see Ivanov, A. S.

Liu, Y., see Li, W.

Lopez, M. F., see Fisher, W. G.

$\mathrm{Lu}$, J., see Li, G.

Lursinsap, C., see Suksawatchon, J.

Lyubetsky, V. A., Pirogov, S. A., Rubanov, L. I. and Seliverstov, A. V., Modeling Classic Attenuation

Regulation of Gene Expression in Bacteria

$1(2007) 155$

Ma, P. C. H. and Chan, K. C. C., An Effective Data Mining

Technique for Reconstructing Gene Regulatory Networks from Time Series Expression Data

3 (2007) 651

Mah, J. T. L. and Chia, K. S., A Gentle Introduction to SNP Analysis: Resources and Tools

5 (2007) 1123

Malik, B. K., see Singh, S.

$1(2007) 135$

Masulis, I. S., see Tutukina, M. N.

2b (2007) 549

Masuya, H., Yoshikawa, S., Heida, N., Toyoda, T., Wakana, S. and Shiroishi, T., PhenoSITE: A Web Database Integrating the Mouse Phenotyping Platform and the Experimental Procedures in Mice

6 (2007) 1173

Mateus, D., Gallois, J.-P., Comet, J.-P. and Le Gall, P., Symbolic Modeling of Genetic Regulatory Networks 2b (2007) 627 Matsumoto, T., see Kaburagi, T.

3 (2007) 669 
Matsunaga, T. and Muramatsu, M., Disease-related Concept

Mining by Knowledge-based Two-Dimensional Gene Mapping

5 (2007) 1047

Mazo, I., see Sivachenko, A. Y.

2b (2007) 429

Mevissen, H. T., see Klinger, R.

6 (2007) 1277

Mezentsev, Y. V., see Ivanov, A. S.

2b (2007) 579

Mikulskis, A., see Fisher, W. G.

5 (2007) 1023

Milbauer, L. C., see Jiang, A.

$4(2007) 875$

Mironova, V. V., see Akberdin, I. R.

2b (2007) 641

Mishchenko, E. L., Bezmaternykh, K. D., Likhoshvai, V. A., Ratushny, A. V., Khlebodarova, T. M., Sournina, N. Yu., Ivanisenko, V. A. and Kolchanov, N. A., Mathematical Model for Suppression of Subgenomic Hepatitis C Virus RNA

Replication in Cell Culture

2b (2007) 593

Mjolsness, E., On Cooperative Quasi-Equilibrium Models of Transcriptional Regulation

Molnar, A. A., see Ivanov, A. S.

Muramatsu, D., see Kaburagi, T.

Muramatsu, M., see Matsunaga, T.

Nguyen, C., Gardiner, K. J. and Cios, K. J., A Hidden Markov Model for Predicting Protein Interfaces

Nurtdinov, R. N., see Ermakova, E. O.

Ogawa, N., see Sakakibara, Y.

Ohno-Machado, L., see Stransky, B.

O'Leary, B. M., Davis, S. G., Smith, M. F., Brown, B., Kemp,

M. B., Almabrazi, H., Grundstad, J. A., Burns, T., Leontiev, V., Andorf, J., Clark, A. F., Sheffield, V. C., Casavant, T. L., Scheetz, T. E., Stone, E. M. and Braun, T. A., Transcript Annotation Prioritization and Screening System (TrAPSS) for Mutation Screening

Olman, V., see Li, G.

Omelyanchuk, L. V., see Gunbin, K. V.

Omelyanchuk, N. A., see Akberdin, I. R.

Ozoline, O. N., see Tutukina, M. N.

2b (2007) 579

3 (2007) 669

5 (2007) 1047

3 (2007) 739

5 (2007) 991

5 (2007) 1103

4 (2007) 977

Ozonov, E. A., see Akberdin, I. R.

Pan, W., see Jiang, A.

Park, T., Kim, K., Yi, S.-G., Kim, J. H., Lee, Y.-S. and Lee, S., Spot Intensity Ratio Statistics in Two-Channel Microarray Experiments

Peng, Y., see Li, W.

2a (2007) 251

Pennington, G., Smith, C. A., Shackney, S. and Schwartz, R.,

Reconstructing Tumor Phylogenies from Heterogeneous

Single-Cell Data

6 (2007) 1155

4 (2007) 817

2b (2007) 491

2b (2007) 641

2b (2007) 549

2b (2007) 641

4 (2007) 875

Pillet, V., see Yip, Y. L. 
Pirogov, S. A., see Lyubetsky, V. A.

$1(2007) 155$

Podkolodny, N. L., see Khlebodarova, T. M.

2b (2007) 507

Polyansky, A. A., Volynsky, P. E. and Efremov, R. G.,

Computer Simulations of Membrane-Lytic Peptides:

Perspectives in Drug Design

2b (2007) 611

Popendorf, K., see Sakakibara, Y.

5 (2007) 1103

Posch, S., Grau, J., Gohr, A., Ben-Gal, I., Kel, A. E. and

Grosse, I., Recognition of CIS-Regulatory Elements with VOMBAT

2b (2007) 561

Qiu, X. and Yakovlev, A., Comments on Probabilistic Models

Behind the Concept of False Discovery Rate

4 (2007) 963

6 (2007) 1233

6 (2007) 1319

Ranganathan, S., see Kanagasabai, R.

2b (2007) 521

2b (2007) 593

Ratushny, A. V., see Mishchenko, E. L.

4 (2007) 839

1 (2007) 31

5 (2007) 1023

Rosenblatt, K. P., see Fisher, W. G.

Rubanov, L. I., see Lyubetsky, V. A.

1 (2007) 155

Sakakibara, Y., Popendorf, K., Ogawa, N., Asai, K. and Sato, K., Stem Kernels for RNA Sequence Analyses

5 (2007) 1103

Sakk, E., On the Computation of Molecular Surface

Correlations for Protein Docking Using

Fourier Techniques

4 (2007) 915

5 (2007) 1139

Salzman, P., see Klebanov, L.

6 (2007) 1277

Sato, K., see Sakakibara, Y.

5 (2007) 1103

6 (2007) 1193

Schaeffer, M., see Shyu, C.-R.

6 (2007) 1155

Scheetz, T. E., see O'Leary, B. M.

6 (2007) 1297

Schwartz, R., see Pennington, G.

2a (2007) 407

Seliverstov, A. V., see Lyubetsky, V. A.

1 (2007) 155

6 (2007) 1261

Sezerman, O. U., see Erdogmus, M.

2a (2007) 407

Sharma, D. K., see Singh, S.

1 (2007) 135

Shavkunov, K. S., see Tutukina, M. N.

2b (2007) 549

Sheffield, V. C., see O'Leary, B. M.

6 (2007) 1155

Shen, C., see Chen, J. Y.

2a (2007) 383

Shiroishi, T., see Masuya, H.

6 (2007) 1173

Shosheva, A., see Kundrotas, P.

3 (2007) 693

Shyr, Y., see Jiang, A.

4 (2007) 875

Shyu, C.-R., Harnsomburana, J., Green, J., Barb, A. S., Kazic, T., Schaeffer, M. and Coe, E., Searching and Mining Visually Observed Phenotypes of Maize Mutants

6 (2007) 1193 
Singh, S., Malik, B. K. and Sharma, D. K., Metabolic Pathway Analysis of S. Pneumoniae: An In Silico Approach Towards

Drug-Design

Sivachenko, A. Y., Yuryev, A., Daraselia, N. and Mazo, I.,

Molecular Networks in Microarray Analysis

Smith, C. A., see Pennington, G.

Smith, M. F., see O'Leary, B. M.

Snir, S., see Eskin, E.

Sournina, N. Yu., see Mishchenko, E. L.

Stepanenko, I. L., see Khlebodarova, T. M.

Stone, E. M., see O'Leary, B. M.

Stransky, B., Barrera, J., Ohno-Machado, L. and de Souza,

S. J., Modeling Cancer: Integration of "Omics" Information in Dynamic Systems

Suksawatchon, J., Lursinsap, C. and Bodén, M., Computing the Reversal Distance Between Genomes in the Presence of Multi-Gene Families via Binary Integer Programming

Sun, S., see Yu, C.

Sung, W.-K., see Han, X.

Tan, M. P., Broach, J. R. and Floudas, C. A., Evaluation of Normalization and Pre-Clustering Issues in a Novel

Clustering Approach: Global Optimum Search with

Enhanced Positioning

Tan, N. C., see Fisher, W. G.

te Boekhorst, R., see Abnizova, I.

Tewari, S., Bhandarkar, S. M. and Arnold, J., Design and

Analysis of an Efficient Recursive Linking Algorithm

for Constructing Likelihood Based Genetic Maps for

a Large Number of Markers

Tikunova, N. V., see Khlebodarova, T. M.

Toyoda, T., see Masuya, $\mathrm{H}$.

Tsiporkova, E. and Boeva, V., Two-Pass Imputation Algorithm for Missing Value Estimation in Gene Expression Time Series

Tutukina, M. N., Shavkunov, K. S., Masulis, I. S. and Ozoline,

O. N., Intragenic Promotor-Like Sites in the Genome of

Escherichia Coli Discovery and Functional Implication

van Berlo, R. J. P., Wessels, L. F. A., de Ridder, D. and

Reinders, M. J. T., Protein Complex Prediction Using

an Integrative Bioinformatics Approach

van de Wiel, M. A., see Bar-Hen, A.

Veuthey, A.-L., see Yip, Y. L.

Volynsky, P. E., see Polyansky, A. A.
1 (2007) 135

2b (2007) 429

2a (2007) 407

6 (2007) 1155

3 (2007) 717

2b (2007) 593

2b (2007) 507

6 (2007) 1155

4 (2007) 977

1 (2007) 117

2a (2007) 297

2a (2007) 281

4 (2007) 895

5 (2007) 1023

2b (2007) 533

2a (2007) 201

2b (2007) 507

6 (2007) 1173

5 (2007) 1005

2b (2007) 549

4 (2007) 839

4 (2007) 987

6 (2007) 1215

2b (2007) 611 
von Rohr, P., Friberg, M. T. and Kadarmideen, H. N.,

Prediction of Transcription Factor Binding Sites Using

Genetical Genomics Methods

3 (2007) 773

Wakana, S., see Masuya, H.

6 (2007) 1173

Walter, K., see Abnizova, I.

2b (2007) 533

Wan, X. and Lin, G., GASA: A Graph-Based Automated NMR

Backbone Resonance Sequential Assignment Program

Wang, J., see Xie, M.

Wang, M., see Chen, J. Y.

Wessels, L. F. A., see van Berlo, R. J. P.

Whiteley, G. R., see Fisher, W. G.

Willman, C., see Andries, E.

Witte, R. and Baker, C. J. O., Towards a Systematic

Evaluation of Protein Mutation Extraction Systems

2a (2007) 313

3 (2007) 795

2a (2007) 383

4 (2007) 839

5 (2007) 1023

1 (2007) 79

6 (2007) 1339

Worth, C. L., Bickerton, G. R. J., Schreyer, A., Forman, J. R.,

Cheng, T. M. K., Lee, S., Gong, S., Burke, D. F. and

Blundell, T. L., A Structural Bioinformatics Approach

to the Analysis of Nonsynonymous Single Nucleotide

Polymorphisms (nsSNPs) and Their Relation

to Disease

6 (2007) 1297

Wu, Y. and Gusfield, D., Efficient Computation of Minimum

Recombination with Genotypes (Not Haplotypes)

Xiao, Y., see Klebanov, L.

2a (2007) 181

5 (2007) 1139

Xie, M., Chen, J. and Wang, J., Research on Parameterized

Algorithms of the Individual Haplotyping Problem

3 (2007) 795

$\mathrm{Xu}, \mathrm{Y}$., see $\mathrm{Li}, \mathrm{G}$.

Yakovlev, A., see Klebanov, L.

4 (2007) 817

5 (2007) 1139

4 (2007) 963

2a (2007) 383

Yan, Z., see Chen, J. Y.

4 (2007) 865

Yi, S.-G., see Park, T.
Yip, Y. L., Lachenal, N., Pillet, V. and Veuthey, A.-L.,

Retrieving Mutation-Specific Information for Human

Proteins in UniProt/Swiss-Prot Knowledgebase

6 (2007) 1215

Yoshikawa, S., see Masuya, H.

Young, N. E., see Fu, Q.

6 (2007) 1173

4 (2007) 937

Yu, C., Lin, Y., Sun, S., Cai, J., Zhang, J., Bu, D., Zhang, Z. and Chen, R., An Iterative Algorithm to Quantify Factors Influencing Peptide Fragmentation During Tandem Mass Spectrometry

2a (2007) 297

Yuryev, A., see Sivachenko, A. Y.

2b (2007) 429

Zhang, J., see $\mathrm{Yu}, \mathrm{C}$.

2a (2007) 297

Zhang, Z., see $\mathrm{Yu}, \mathrm{C}$.

2a (2007) 297 